\title{
Review Article \\ Phononic First Band Gap of Quaternary Layered Periodic Structure with the Lumped-Mass Method
}

\author{
Chen Yuan, ${ }^{1}$ Li Jing, ${ }^{1}$ Zhou Jingdong, ${ }^{1}$ Huang Tao, ${ }^{1}$ Zhou Minggang, ${ }^{1}$ and Yu Dao Yuan ${ }^{2}$ \\ ${ }^{1}$ Hubei Automotive Structural Engineering Center of Noise \& Vibration, Hubei University of Technology, Wuhan 430068, China \\ ${ }^{2}$ The Institute of Mechanical Science and Engineering, Huazhong University of Science and Technology, Wuhan 430074, China \\ Correspondence should be addressed to Chen Yuan; 57251966@qq.com
}

Received 20 January 2013; Accepted 5 February 2014; Published 27 February 2014

Academic Editor: Nuno Maia

Copyright (c) 2014 Chen Yuan et al. This is an open access article distributed under the Creative Commons Attribution License, which permits unrestricted use, distribution, and reproduction in any medium, provided the original work is properly cited.

\begin{abstract}
Existing band gap analysis is mostly focused on the binary structure, while the researches on the quaternary layered periodic structure are still lacking. In this paper, the unidimensional lumped-mass method in the phonic crystal theory is firstly improved so that the material viscoelasticity can be taken into consideration. Then, the binary layered periodic structure is converted into a quaternary one and band gaps appear at low frequency range. Finally, the effects of density, elastic modulus, damping ratio, and the thickness of single material on the first band gap of the quaternary layered periodic structure are analyzed after the algorithm is promoted. The research findings show that effects of density, elastic modulus, and thickness of materials on the first band gap are considerable but those of damping ratio are not so distinct. This research provides theoretical bases for band gap design of the quaternary layered periodic structure.
\end{abstract}

\section{Introduction}

In recent years, the researches on energy band features of periodic structure constructed artificially based on the phononic crystal theory have drawn more and more attention. This is because artificially constructed periodic structure is characterized by bandpass and bandstop of classical waves. In the acoustics, the gaps between energy bands are called phononic band gaps and the corresponding periodic structure is called phononic crystal. As early as in the 1980s, Achenbach and Kitahara $[1,2]$ studied the propagation of elastic waves in periodic medium with spherical cavity. Sigalas and Economou [3] first theoretically demonstrated that band gaps appeared in periodic lattice structure formed by embedding solid spherical materials into the matrix. Afterwards, the researchers [4-6] studied the influence of material properties on phononic band gaps and found that the main reason why phononic band gaps occurred lied in periodic variation of material properties like the density and elastic modulus.

The layered periodic structure, formed by two or more types of materials that are alternatively arranged, belongs to unidimensional phononic crystal and has attracted wide attention of domestic and overseas scholars. Esquivel-Sirvent and Cocoletzi [7] deduced the reflection coefficient and chromatic dispersion relation for the propagation of elastic waves in layered periodic structure. Hussein et al. $[8,9]$ studied the layered periodic structure constructed with two types of materials and obtained the band gap features in the variation of material distribution in each period. Meanwhile, they observed the vibration of the corresponding finite periodic structure and the results showed that the damping features could be fully embodied when the number of periods reached a certain value. Zhao and Wei [10] studied unidimensional viscoelastic phononic crystal and presented band gaps with different viscoelastic coefficient and relaxation time, the result of which indicated that usage of viscoelastic materials could lead to changes in position and width of band gaps. $\mathrm{Wu}$ et al. [11] used functionally graded materials as the connection layer in unidimensional layered phononic crystal and discussed the effects of the introduction of functionally graded materials on band gaps. Apart from material properties, it was found that structural parameters also affected the position and width of band gaps of phononic crystals. Sesion Jr. et al. 


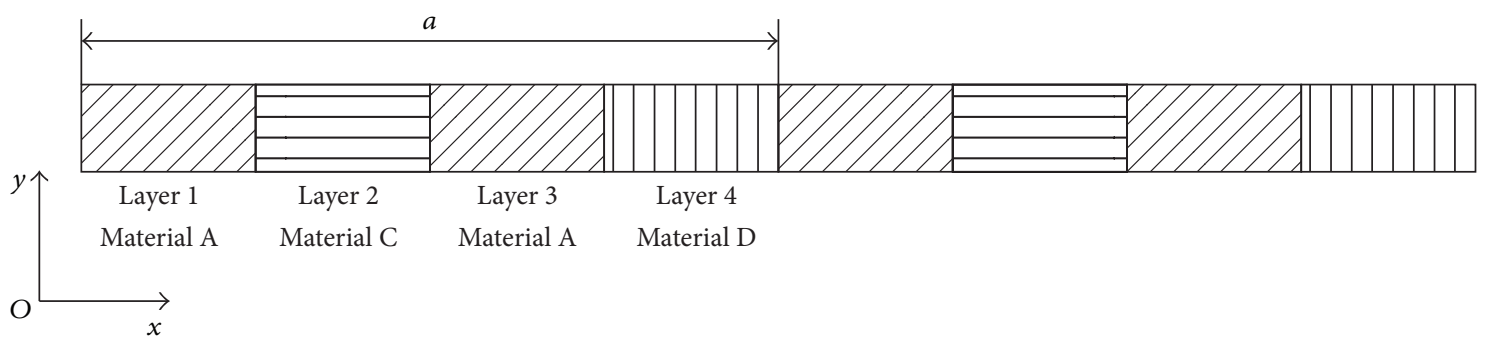

FIGURE 1: A quaternary layered periodic structure.

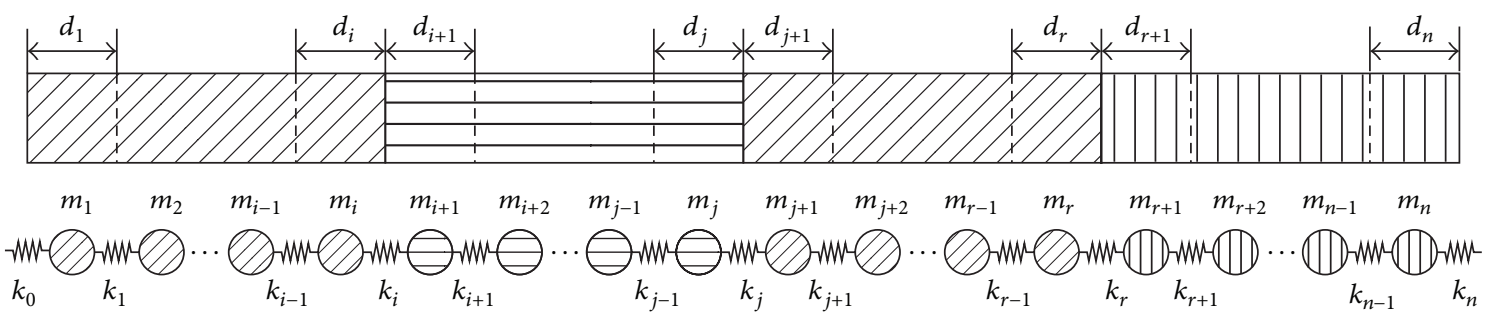

FIGURE 2: Model for the simplification of a quaternary layered periodic structure into a mass-spring structure.

[12] studied band gaps of piezoelectric Fibonacci superlattice structure and succeeded in controlling phononic filtering with this type of semiconductor. Li et al. [13-16] studied the effects of random disorder on band gaps in layered phononic crystal structure and found that localization degree went up apparently and band gap narrowed with the increase of randomness. Policarpo et al. [17], in the research on binary rod periodic structure, performed the modal analysis, harmonic response analysis, and energy band calculation and compared these three methods. Through adjusting the proportion of materials in each period, the position of first band gap changed and appeared at low frequency range.

In unidimensional periodic structure, gaps in the elastic band structure have been predicted using a variety of computational methods such as plane-wave expansion and transfer matrix. Jensen [18] calculated periodic mass-spring structure and confirmed band gaps appeared in both unidimensional and bidimensional periodic mass-spring structures. Wang et al. [19] introduced the discretization method of vibration mechanics into band gap computation of solidsolid phononic crystal and transformed a continuous system problem into a discrete problem by concentrating mass of continuous medium on finite nodes and representing rigidity of continuous medium with lines between nodes. This method is called lumped mass method, and it provides a convenient setting for the realization and programming.

In specific application, Wang et al. [19] achieved the purpose of vibration isolation through researches on band gap features of gear shaft of periodic phononic crystal structure. Richards and Pines [20] studied flexural wave propagation characteristics of periodic suspension spring beam structure and obtained locally resonance band gaps, which were further proved in experiments and found a wide application in practical projects, like engine and dynamic vibration absorber.
Despite the fact that researches on phononic crystal have enjoyed remarkable development, band gap analysis on layered polynary periodic structure is still limited. In this paper, the quaternary layered periodic structure is first simplified into a unidimensional phononic crystal structure according to the phononic crystal theory, and then the effects of density, elastic modulus, damping ratio, and thickness proportion of each type of material on the first band gap are analyzed with the lumped-mass method.

\section{Theoretical Modeling of Quaternary Layered Periodic Structure}

Figure 1 shows that three types of materials are arranged in an order of ACAD along the direction Ox to constitute the quaternary layered periodic structure.

A layered structure, if its dimension along the aperiodic direction (the length and width of layered periodic structure) is far greater than the wavelength of frequency range that considered, can be treated as a unidimensional phononic crystal structure. In consideration of the propagation of plane waves along the direction $\mathrm{Ox}$ and the plane waves are longitudinal, the vibration of layered periodic structure is similar to the vibration of periodic mass-spring structure. Each period is divided into several pieces with the lumpedmass method. Those pieces are deemed as mass points and two adjacent mass points are connected with a spring that has rigidity but no mass. Consequently, a unidimensional phononic crystal is simplified into a periodic mass spring structure, as shown in Figure 2.

For viscoelastic materials, there exists damping coefficient $c_{j}$ besides rigidity $k_{j}$ between mass points in the lumped-mass model, as shown in Figure 3. 


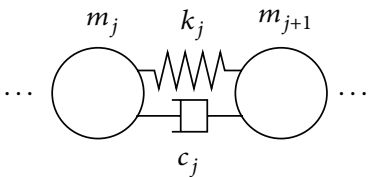

FIGURE 3: Mass-spring with damping force.

After a unidimensional phononic crystal structure is simplified into a periodic mass-spring structure with the lumpedmass method, it can be found that movement of each mass point only relates to two mass points on the left and right. According to Newton's second law, the dynamic differential equation for the system can be written as

$$
\begin{aligned}
m_{j} \ddot{x}_{j}= & k_{j}\left(x_{j+1}-x_{j}\right)-k_{j-1}\left(x_{j}-x_{j-1}\right) \\
& +c_{j}\left(\dot{x}_{j+1}-\dot{x}_{j}\right)-c_{j-1}\left(\dot{x}_{j}-\dot{x}_{j-1}\right), \quad j=1, \ldots, n,
\end{aligned}
$$

where $m_{j}$ denotes mass of the $j$ th mass point, $k_{j-1}$ and $k_{j}$ are the rigidity of two springs before and after the $j$ th mass point, respectively, $c_{j-1}$ and $c_{j}$ are damping coefficient before and after the $j$ th mass point, $x_{j-1}, x_{j}$, and $x_{j+1}$ are the movement of the $(j-1)$ th, $j$ th, and $(j+1)$ th mass points, $\dot{x}_{j-1}, \dot{x}_{j}$ and $\dot{x}_{j+1}$ are the velocity of the $(j-1)$ th, $j$ th, and $(j+1)$ th mass points, and $\ddot{x}_{j}$ is the acceleration of the $j$ th mass point.

The mass of the $j$ th mass point is

$$
m_{j}=\rho_{j} S d_{j} .
$$

Here, $\rho_{j}$ and $d_{j}$ represent the density and the length of the unit corresponding to the $j$ th mass point, and $S$ refers to cross section area of the layered structure.

When two adjacent mass points are of the same material, the rigidity between mass points is shown in Figure 4 .

The relation between normal stress and the displacement along the direction $x$ is

$$
\frac{F_{j}^{x}}{S}=\left(\lambda_{A}+2 \mu_{A}\right) \frac{\Delta x_{j}}{d_{j} / 2+d_{j+1} / 2},
$$

where $\lambda_{A}$ and $\mu_{A}$ refer to Lamé constants of material A, $\Delta x_{j}$ is displacement of the $j$ th mass point along the direction $x$, $d_{j} / 2+d_{j+1} / 2$ is original length of spring, and $S$ refers to cross section area of the layered structure.

Lamé constants can be calculated by

$$
\begin{gathered}
\lambda_{A}=\frac{E_{A} \sigma_{A}}{\left(1+\sigma_{A}\right)\left(1-2 \sigma_{A}\right)}, \\
\mu_{A}=\frac{E_{A}}{2\left(1+\sigma_{A}\right)} .
\end{gathered}
$$

$E_{A}$ is the elastic modulus of material A and $\sigma_{A}$ is Poisson's ratio of material $\mathrm{A}$.

Hence, the rigidity is

$$
k_{j}=\frac{2\left(\lambda_{A}+2 \mu_{A}\right) S}{d_{j}+d_{j+1}} .
$$
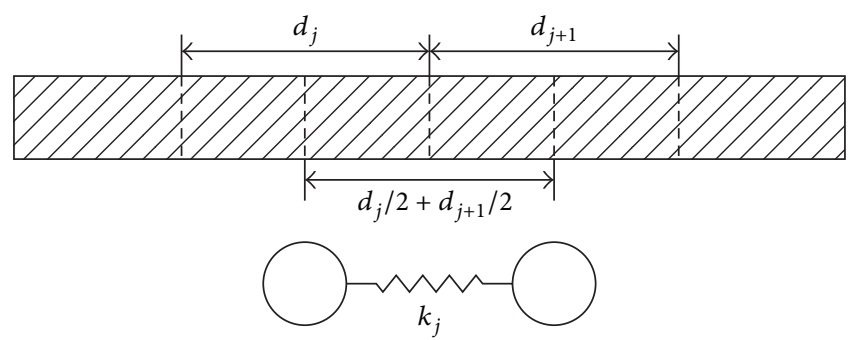

FIGURE 4: The simplified model for rigidity among mass points of the same material.

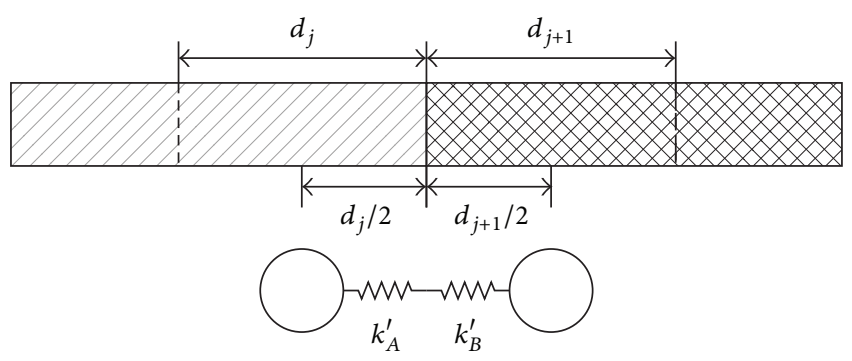

FIGURE 5: The simplified model for rigidity among mass points of different materials.

When two adjacent mass points are of different materials, the rigidity between mass points is deemed as series connection of springs of different rigidities, as shown in Figure 5.

The relation between normal stress and the displacement along the direction $x$ is

$$
\begin{aligned}
\frac{F_{j}^{x}}{S}=\left(\lambda_{A}+2 \mu_{A}\right) \frac{\Delta x_{j}^{A}}{d_{j} / 2}, & k_{j}^{A}=\left(\lambda_{A}+2 \mu_{A}\right) \frac{S}{d_{j} / 2}, \\
\frac{F_{j}^{x}}{S}=\left(\lambda_{B}+2 \mu_{B}\right) \frac{\Delta x_{j}^{B}}{d_{j+1} / 2}, & k_{j}^{B}=\left(\lambda_{B}+2 \mu_{B}\right) \frac{S}{d_{j+1} / 2} .
\end{aligned}
$$

Therefore, the rigidity between two types of materials is

$$
k_{j}=\frac{k_{j}^{A} k_{j}^{B}}{k_{j}^{A}+k_{j}^{B}}=\frac{2\left(\lambda_{A}+2 \mu_{A}\right)\left(\lambda_{B}+2 \mu_{B}\right) s}{\left(\lambda_{A}+2 \mu_{A}\right) d_{j+1}+\left(\lambda_{B}+2 \mu_{B}\right) d_{j}} .
$$
$\zeta_{j}$,

Since damping coefficient $c_{j}$ is related with damping ratio

$$
c_{j}=2 \cdot \zeta_{j} \sqrt{m_{j} k_{j}} .
$$

Here, it must be pointed out that the calculation of damping coefficient $c_{j}$ into two casesis similar to the calculation of rigidity $k_{j}$. The damping between two mass points of the same material can be calculated directly according to (8). For the damping between two mass points of different materials, (8) is approximately applicable since it only exists at the junction of materials after the discretization of each cell. 
TABLE 1: Material parameters.

\begin{tabular}{lcccccc}
\hline Material code & Material name & Density $\rho\left(\mathrm{kg} \cdot \mathrm{m}^{-3}\right)$ & Elastic modulus $E(\mathrm{~Pa})$ & Poisson's ratio $\sigma$ & Thickness $d(\mathrm{~m})$ & Damping ratio $\zeta$ \\
\hline A & Silicone Rubber & 1300 & $1.175 e 5$ & 0.4688 & 0.00125 \\
$\mathrm{C}$ & Steel & 7780 & $2.106 e 11$ & 0.3 & 0.00125 & 0.1 \\
$\mathrm{D}$ & Tungsten & 19100 & $3.54 e 11$ & 0.3505 & 0.00125 & 0 \\
\hline
\end{tabular}

According to Bloch Theorem, the solution of motion equation of mass point can be assumed as simple harmonic vibration with amplitude $A_{j}^{\prime}$ and angular frequency $\omega$ under periodic boundary conditions.

Consider

$$
x_{j}=A_{j}^{\prime} e^{i\left(q \sum_{j=1}^{j} d_{j}-\omega t+\varphi(j)\right)}=A_{j} e^{i\left(q \sum_{j=1}^{j} d_{j}-\omega t\right)},
$$

where $q \sum_{j=1}^{j} d_{j}$ denotes phase factor of the $j$ th mass point. $q$ stands for lattice wave vector and it is in the first Brillouin zone. $\varphi(j)$ indicates the phase difference caused by the damping and it is a constant value when the damping is certain. Therefore, $\varphi(j)$ is incorporated in the amplitude $A_{j}^{\prime}$ and the amplitude of mass point becomes $A_{j}$.

When (9) is substituted into dynamic differential equation system (1), a simple linear equation can be obtained after simplification.

Consider

$$
\begin{aligned}
& -\omega^{2} m_{j} A_{j}-i \omega \\
& \quad \times\left[-c_{j} e^{i q d_{j+1}} A_{j+1}+\left(c_{j}+c_{j-1}\right) A_{j}-c_{j-1} e^{-i q d_{j-1}} A_{j-1}\right] \\
& +\left[-k_{j} e^{i q d_{j+1}} A_{j+1}+\left(k_{j}+k_{j-1}\right) A_{j}-k_{j-1} e^{-i q d_{j-1}} A_{j-1}\right]=0 .
\end{aligned}
$$

In (10), when $j=0$ or $n$, periodic boundary conditions should be added since parameters in the periods before and after are employed. For the periodic structure, the first element of $j$ th cell is the same as the first element of the $j+1$ th cell, and the last element of $j$ th cell is the same as the last element of the $j-1$ th cell. The periodic boundary conditions can be applied as follows:

$$
\begin{array}{cc}
k_{0}=k_{n}, & k_{1}=k_{n+1}, \\
m_{0}=m_{n}, & m_{1}=m_{n+1}, \\
c_{0}=c_{n}, & c_{1}=c_{n+1}, \\
d_{0}=d_{n}, & d_{1}=d_{n+1}, \\
A_{0}=A_{n}, & A_{1}=A_{n+1} .
\end{array}
$$

When periodic boundary conditions (11) are substituted into linear equation (10), the linear equation system can be presented in the form of a matrix.

Consider

$$
\left(-\omega^{2} \mathbf{M}-i \omega \mathbf{C}(q)+\mathbf{K}(q)\right) \mathbf{A}=0 .
$$

Since there are both $i \omega$ and $\omega^{2}$ in the linear equation system (12), it is not a standard eigenvalue equation. For this reason, further mathematical treatment is necessary before energy band structure is obtained.

$$
\text { If } \lambda=i \omega
$$

$$
\begin{gathered}
\lambda^{2}=-\omega^{2}, \\
\mathbf{B}=i \omega \mathbf{A}=\lambda \mathbf{A} .
\end{gathered}
$$

When (13) and (14) are substituted into matrix equation (12),

$$
\begin{aligned}
& -\omega^{2} \mathbf{M A}-i \omega \mathbf{C}(q) \mathbf{A}+\mathbf{K}(q) \mathbf{A} \\
& =\lambda \mathbf{M B}-\mathbf{C}(q) \mathbf{B}+\mathbf{K}(q) \mathbf{A}=0 .
\end{aligned}
$$

Equation (15) can be simplified into

$$
\mathbf{M}^{-1} \mathbf{C}(q) \mathbf{B}-\mathbf{M}^{-1} \mathbf{K}(q) \mathbf{A}=\lambda \mathbf{B} .
$$

When (14) and (16) are put together,

$$
\left[\begin{array}{cc}
\mathbf{0} & \mathbf{I} \\
-\mathbf{M}^{-1} \mathbf{K} & \mathbf{M}^{-1} \mathbf{C}
\end{array}\right]\left[\begin{array}{l}
\mathbf{A} \\
\mathbf{B}
\end{array}\right]=\lambda\left[\begin{array}{l}
\mathbf{A} \\
\mathbf{B}
\end{array}\right] .
$$

Through the solution of eigenvalue $\lambda$ of matrix $\left[\begin{array}{cc}\mathbf{0} & \mathbf{I} \\ -\mathbf{M}^{-1} \mathbf{K} \mathbf{M}^{-1} \mathbf{C}\end{array}\right]$, corresponding angular frequency $\omega(q)$ is obtained. Energy band diagram of elastic wave in a unidimensional phononic crystal structure can be drawn since wave vector $q$ and corresponding eigenvalue $\omega(q)$ are determined.

\section{Results and Analysis}

In this paper, it is supposed that materials $\mathrm{A}, \mathrm{C}$, and D are arranged in an increasing order of the density and elastic modulus in each period of ABCD structure. However, the materials are alternatively arranged in each period of ACAD structure, which is similar to the binary periodic structure constructed with materials $\mathrm{A}$ and $\mathrm{C}$.

In fact, AC structure can also be deemed as the quaternary layered periodic structure, with the lattice constant of $2 a$ and the materials arranged in ACAC sequence in each period. Therefore, the structures (a) and (b) shown in Figure 6 are essentially the same. The algorithm mentioned above can be employed to calculate the energy band curves of these three structures and necessary material parameters are as shown in Table 1.

According to the energy band curves of these three structures, it can be found in Figure 7 that the band gaps occur 


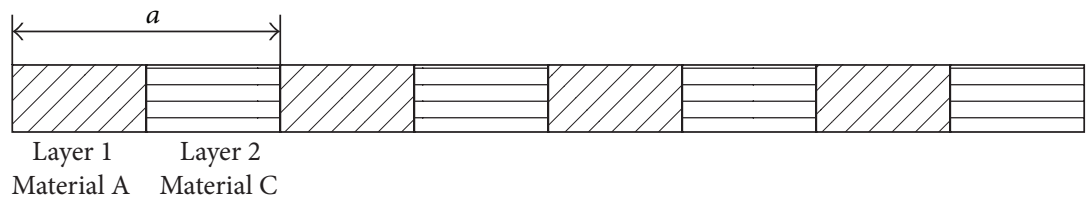

(a)

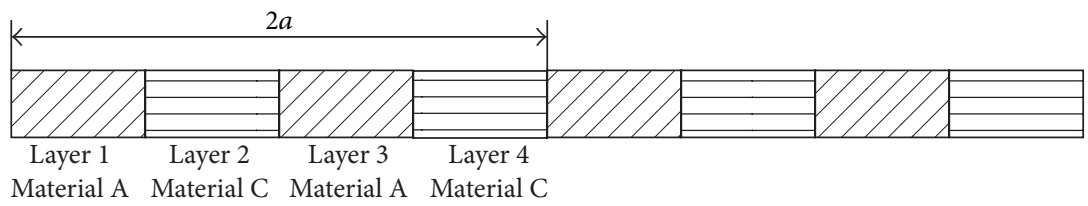

(b)

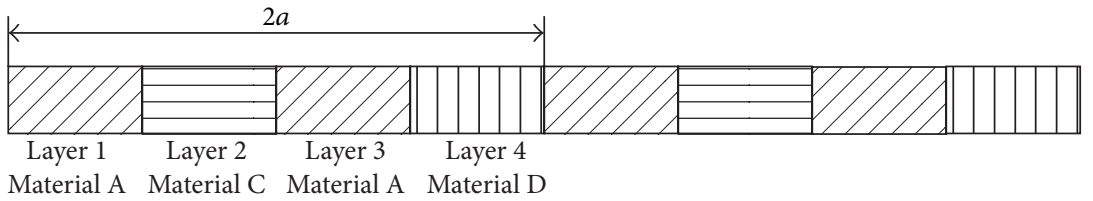

(c)

FIgURE 6: Three types of layered periodic structure.

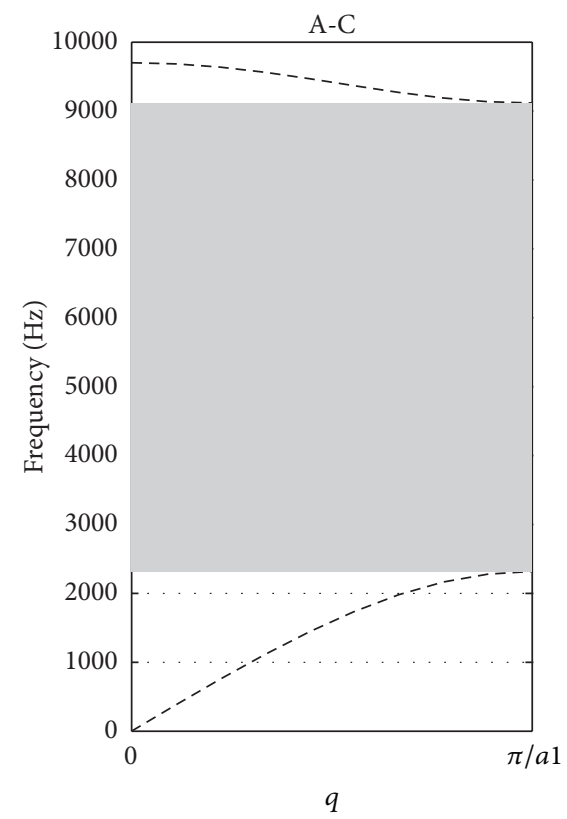

(a)

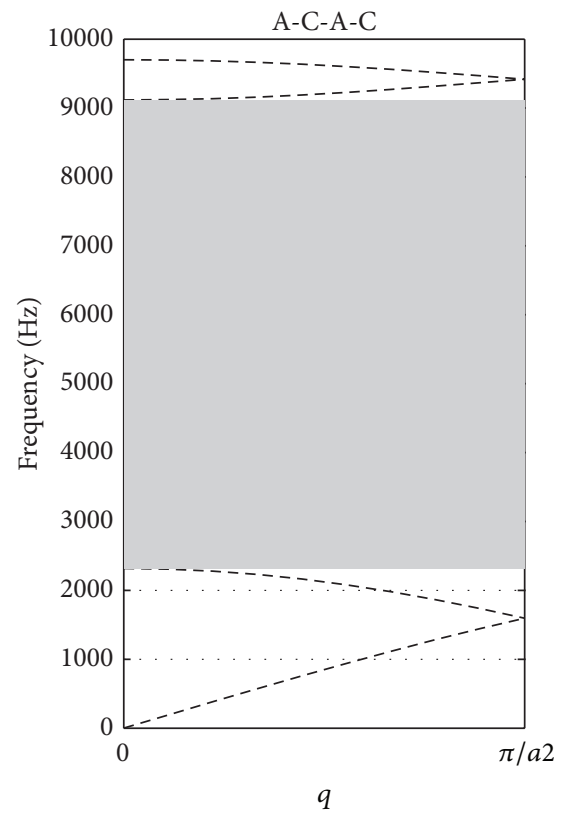

(b)

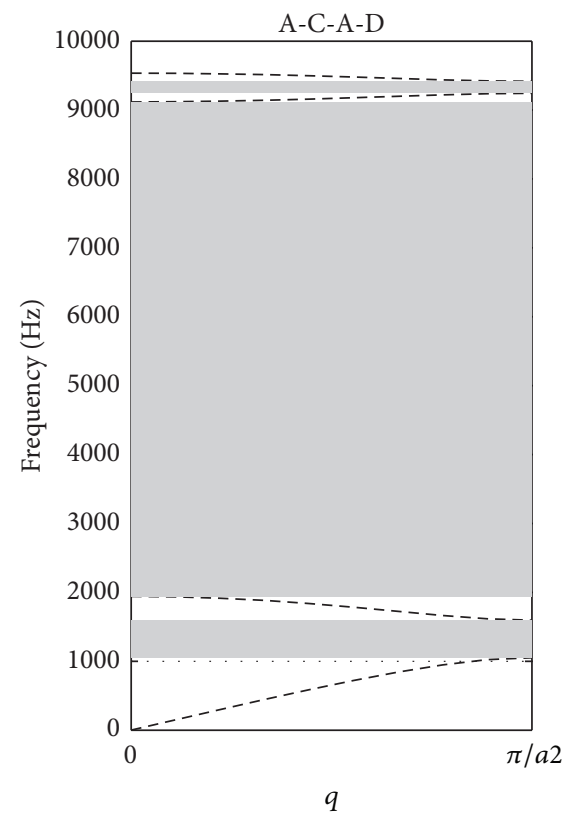

(c)

FIGURE 7: Energy band of three types of layered periodic structure.

at similar frequency range in spite of the changes of the energy band curves. The change of lattice constant causes the alternation of the first Brillouin zone as well as the value range of wave vector, thus giving rise to the changes of energy band curves. In ACAC structure and ACAD structure, the first Brillouin zones are the same because of the same lattice constant, but the energy band curves have altered and the new band gaps have come into being since the material in the fourth layer is changed.
The effects of density, elastic modulus, damping ratio, and thickness variation of materials on the first band gap of the quaternary layered periodic structure constructed with three materials, namely ACAD structure, are discussed below.

3.1. Effect of Density on Band Gap. According to the algorithm presented above, the damping force is ignored and the material parameters are as shown in Table 1 when the effect of material density on the first band gap is studied. 


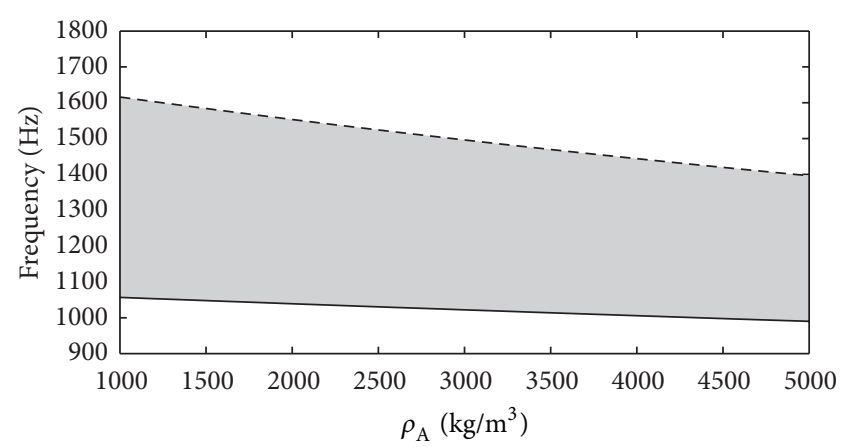

(a)

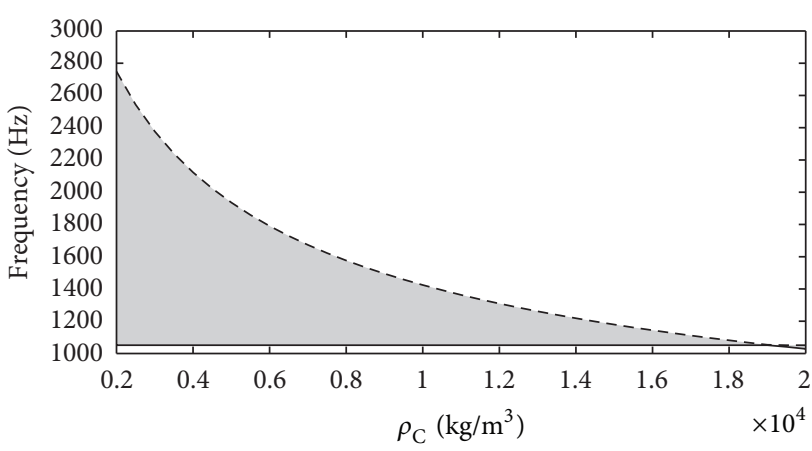

(b)

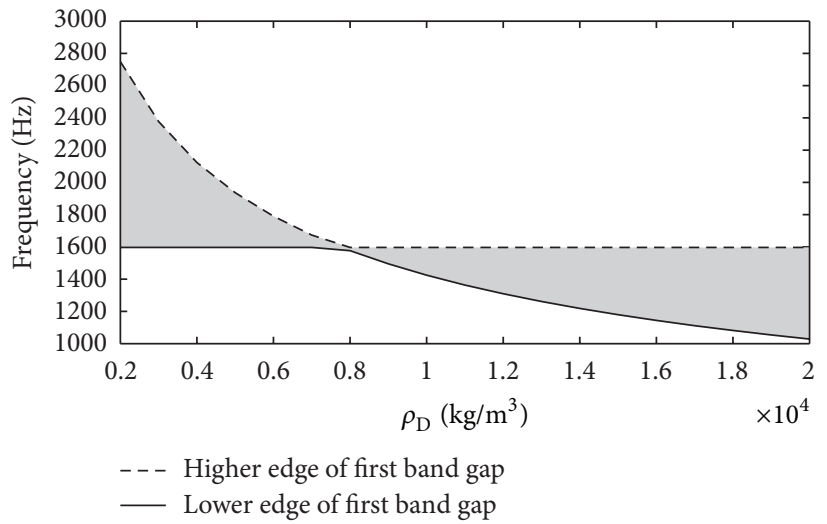

(c)

FIGURE 8: Effects of the density on the first band gap in ACAD structure.

Figures $8(\mathrm{a}), 8(\mathrm{~b})$, and $8(\mathrm{c})$ show the relation between density of each type of material and the first band gap, respectively, in ACAD structure.

It can be seen from Figure 8(a) that the lower edge and the higher edge of the first band gap both fall and the band gap narrows when the density of material $\mathrm{A}$ increases from $1000 \mathrm{~kg} \cdot \mathrm{m}^{-3}$ to $5000 \mathrm{~kg} \cdot \mathrm{m}^{-3}$.

It can be observed from Figure 8(b) that the higher edge of the first band gap decreases but the lower edge of the first band gap remains unchanged, and band gap width decreases when the density of material C increases from $2000 \mathrm{~kg} \cdot \mathrm{m}^{-3}$ to $19000 \mathrm{~kg} \cdot \mathrm{m}^{-3}$. On the contrary, when density of material $\mathrm{C}$ increases from $19000 \mathrm{~kg} \cdot \mathrm{m}^{-3}$ to $20000 \mathrm{~kg} \cdot \mathrm{m}^{-3}$, the higher edge of the first band gap stays the same but the lower edge of the first band gap begins to decrease, and band gap width increases.

From Figure 8(c), we can see that the higher edge of the first band gap falls but the lower edge of the first band gap remains the same, and band gap width decreases when the density of material D increases from $2000 \mathrm{~kg} \cdot \mathrm{m}^{-3}$ to $8000 \mathrm{~kg} \cdot \mathrm{m}^{-3}$. However, the higher edge of the first band gap is fixed but the lower edge of the first band gap decreases, and band gap becomes wider when the density of material $\mathrm{D}$ increases from $8000 \mathrm{~kg} \cdot \mathrm{m}^{-3}$ to $20000 \mathrm{~kg} \cdot \mathrm{m}^{-3}$.

In comparison of Figures $8(\mathrm{~b})$ and $8(\mathrm{c})$, it can be found that the band gaps decrease and even disappear when the density of two materials is closer.
3.2. Effect of Elastic Modulus on Band Gap. Figure 9 presents the relation between elastic modulus of three types of materials in Figures 9(a), 9(b), and 9(c), respectively. The effect of damping force is ignored and other material parameters are as shown in Table 1.

From Figure 9(a), we can observe that the lower edge and the higher edge of the first band gap both rise and band gap width increases when elastic modulus of material A increases.

It can be seen from Figure 9(b) that the lower edge and the higher edge of the first band gap both rise but begin to converge gradually, and band gap width decreases slightly when elastic modulus of material C increases.

It can be observed from Figure 9(c) that the lower edge and the higher edge of the first band gap both increase but begin to converge gradually, and band gap becomes wider with the growth of elastic modulus of material D.

The elastic modulus of different materials has different effects on the first band gap. For material $\mathrm{A}$, with the increase of the difference of elastic modulus between materials $\mathrm{A}$ and adjacent materials $\mathrm{C}$ and $\mathrm{D}$, the first band gap occurs at high frequency range. For materials $\mathrm{C}$ and $\mathrm{D}$, when their elastic modulus reaches a certain extent and elastic modulus of material A is absolutely low compared with those of materials $\mathrm{C}$ and $\mathrm{D}$, the further increase of elastic modulus of material $\mathrm{C}$ and $\mathrm{D}$ does not bring about any effect on the first band gap.

3.3. Effect of Damping Ratio on Band Gap. Figure 10 displays the effect of damping ratio of material $\mathrm{A}$ on the first band 


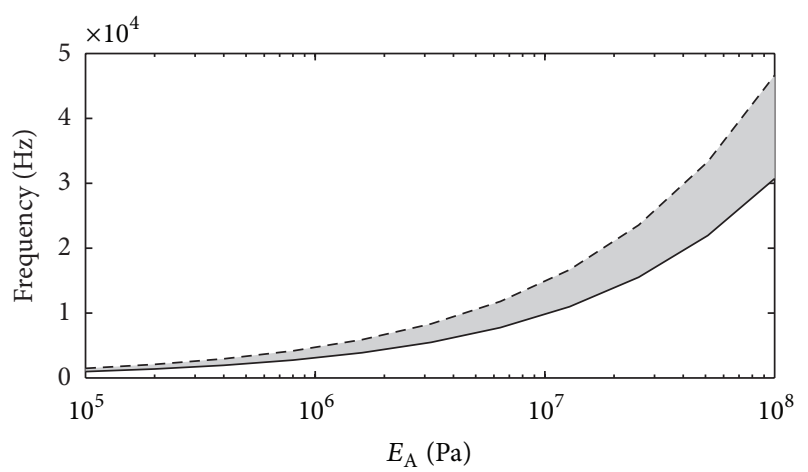

(a)

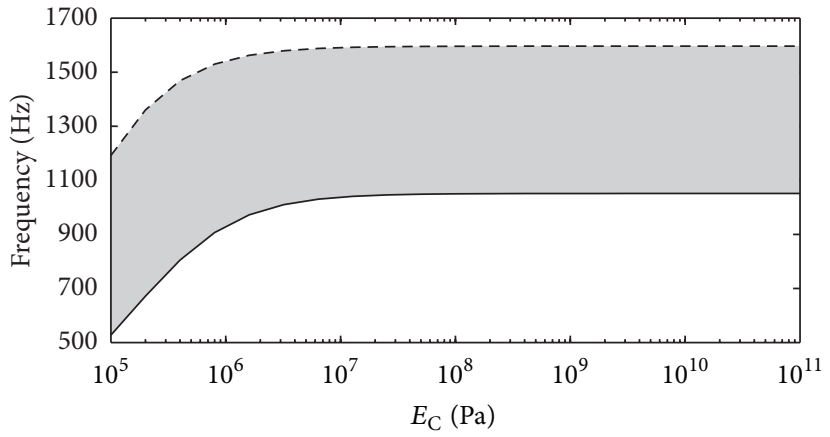

(b)

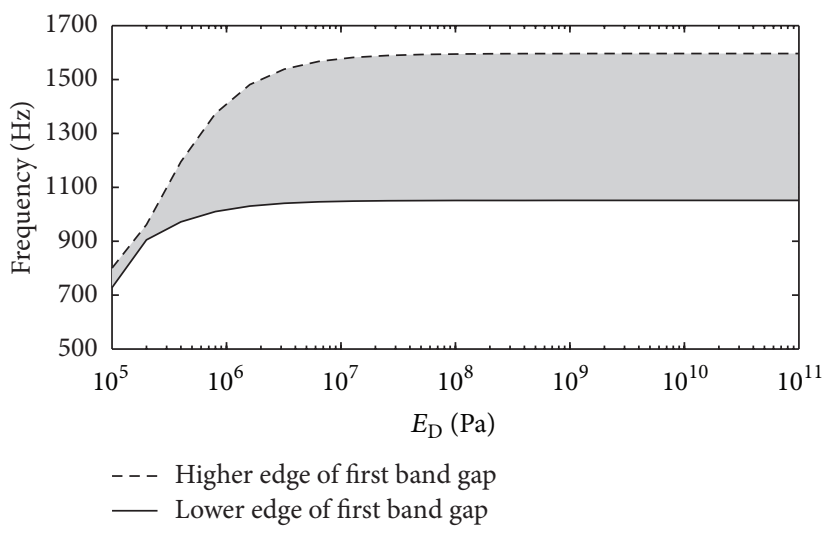

(c)

FIGURE 9: Effects of elastic modulus on the first band gap in ACAD structure.

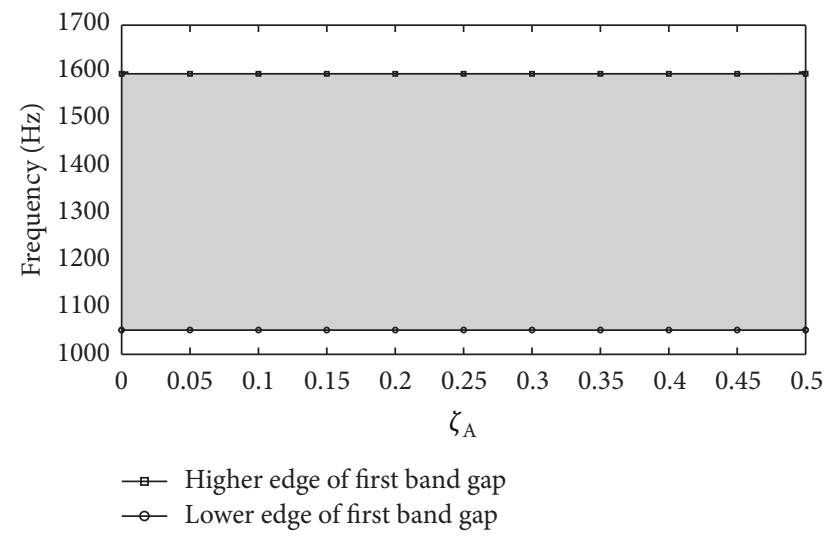

Figure 10: Effect of damping coefficient of material A on the first band gap in ACAD structure.

gap in ACAD structure and necessary material parameters are as shown in Table 1. The lower edge and the higher edge of the first band gap both remain unchanged when the damping ratio of material A increases from 0 to 0.5 . This indicates that band gap won't be affected if viscoelasticity of materials is simply represented by damping ratio.

3.4. Effect of Material Thickness on Band Gap. In ACAD structure, on the premise of unchanged lattice constant $a$, the thickness of one layer in a period is increased from $0.1 a$ to $0.7 a$ and the thickness of the other three layers is reduced from $0.3 a$ to $0.1 a$ so as to observe the alternation of thickness of one layer on the first band gap. Since the first and third layers are both of material A, the effect of thickness alternation of the first, second, and fourth layers on band gap will be discussed.

Figures 11(a), 11(b), and 11(c) show the relation curve between material thickness proportion of the first, second, and fourth layers and the first band gap in a period in ACAD structure, respectively.

It can be seen from Figure 11(a) that the lower edge of the first band gap fluctuates in a very small range with the growth of thickness of the first layer. When the thickness proportion of the first layer increases from $10 \%$ to $25 \%$, both the higher edge of the first band gap and band gap width decrease; when the thickness proportion of the first layer increases from $25 \%$ to $70 \%$, the higher edge of the first band gap and the band gap width both show the opposite trend. This shows that the smallest band gap appears when the proportion of material A is similar to that of other materials and the growing width of the first band gap is witnessed whether the proportion of material $\mathrm{A}$ is further increased or decreased.

It can be observed from Figure 11(b) that the lower edge of the first band gap increases but the higher edge of the first band gap decreases, and band gap becomes narrower when the thickness proportion of the second layer increases from 


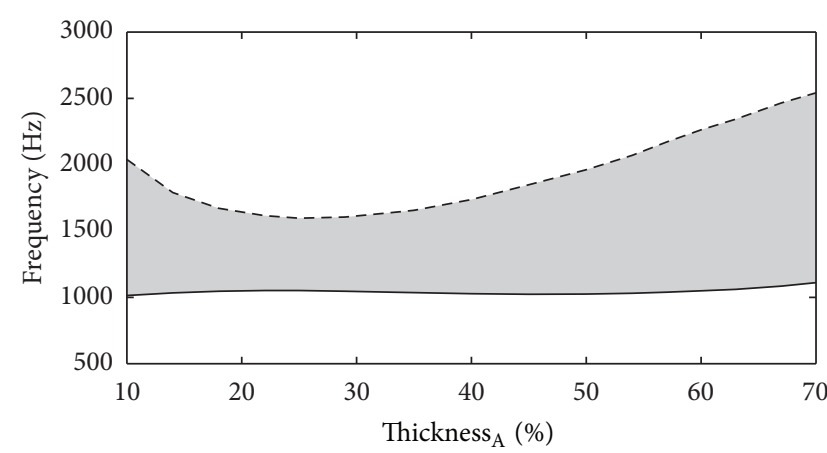

(a)

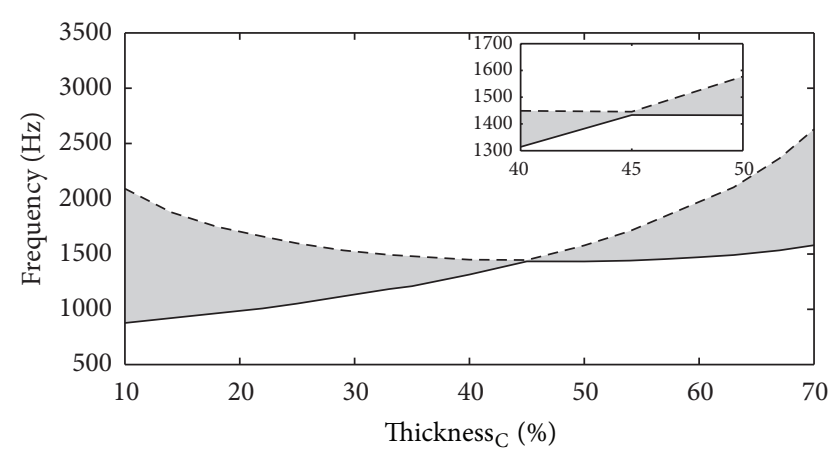

(b)

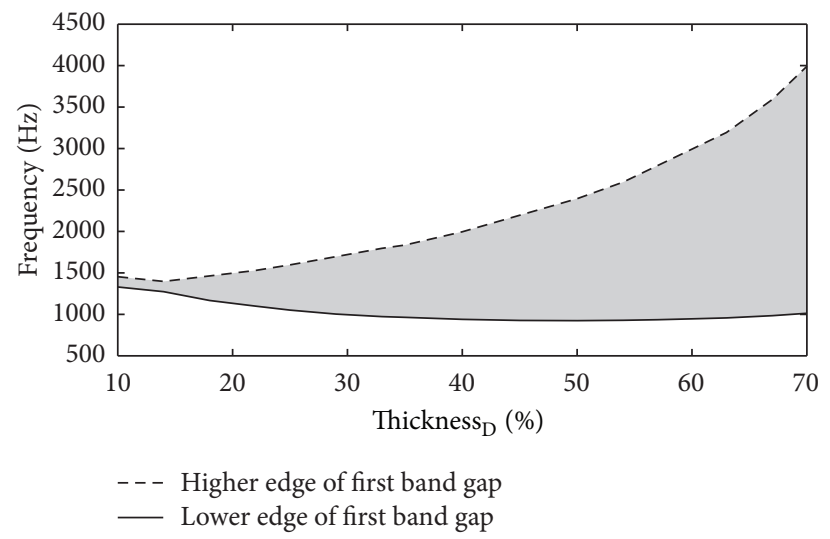

(c)

FIGURE 11: Effects of thickness proportion of materials on the first band gap in ACAD structure

$10 \%$ to $45 \%$. However, the lower edge of the first band gap still goes up gradually and the higher edge of the first band gap increases, and the band gap becomes wider when thickness proportion of the second layer increases from $45 \%$ to $70 \%$. It must be pointed out that the mass of materials $\mathrm{C}$ and $\mathrm{D}$ are equal when their thickness account for $45 \%$ and $18.3 \%$, respectively. At this time, ACAD structure resembles ACAC structure, so the band gaps decrease.

From Figure 11(c), we can see that the lower edge of the first band gap decreases gradually when the thickness proportion of the fourth layer increases from $10 \%$ to $50 \%$ but begins to go up when the thickness proportion of the fourth layer increases from $50 \%$ to $70 \%$; the lower edge of the first band gap decreases when the thickness proportion of the fourth layer increases from $10 \%$ to $15 \%$ but increases gradually when the thickness proportion of the fourth layer increases from $15 \%$ to $70 \%$. The band gap width increases with the growth of thickness proportion of the fourth layer.

Hence, effects on the first band gap will be different as the thickness proportion of each layer of material is changed.

\section{Conclusions}

In this paper, the binary layered periodic structure constructed with materials $\mathrm{A}$ and $\mathrm{C}$ is modified to the quaternary layered periodic structure constructed with materials $\mathrm{A}, \mathrm{C}$, and $\mathrm{D}$ (i.e., ACAD Structure), thus, the energy band curve alters and the band gaps occur at lower frequency range. The effects of density, elastic modulus, damping ratio, and thickness proportion of materials on band gaps are studied. The research findings are as follows.

(1) As for the material properties, the density is the most influential factor, followed by the elastic modulus. The viscoelasticity of materials is deemed as the damping and has little effect on the first band gap.

(2) For low-density material A, the lower edge and the higher edge of the first band gap both fall and the band gap width decreases with the growth of density. For high density materials $\mathrm{C}$ and $\mathrm{D}$, when their density contrast decreases, the higher edge of the first band gap falls but the lower edge remains unchanged; namely, the band gap width decreases. On the contrary, when their density contrast increases, the higher edge of the first band gap stays the same but the lower edge falls. The increase of density contrast results in the growing width of the first band gap.

(3) For low hardness material A, the lower edge and the higher edge of the first band gap increase at the same time with the growth of elastic modulus; namely, the width of the first band gap expands. So it is with highhardness materials $\mathrm{C}$ and $\mathrm{D}$. 
(4) When the proportion of materials is changed, the effects on the first band gap change as well. The smallest first band gap appears when the proportion of low-hardness and low-density materials is similar to that of high-hardness and high-density materials. Whether the proportion of low-hardness and lowdensity materials is increased or not, the first band gap becomes wider. However, when the thickness of material $\mathrm{C}$ is changed and the mass of material $\mathrm{C}$ is equal to that of material $\mathrm{D}$ in each period, the first band gap becomes narrower and even disappears. The expanding mass difference of two materials leads to the wider first band gap.

\section{Conflict of Interests}

The authors declare that there is no conflict of interests regarding the publication of this paper.

\section{Acknowledgments}

The authors wish to express their gratitude for Grant no. 50975081 from the National Natural Science Foundation of China and Grant no. 2011CDB085 from the Natural Science Foundation of Hubei Province which made this research possible.

\section{References}

[1] J. D. Achenbach and M. Kitahara, "Reflection and transmission of an obliquely incident wave by an array of spherical cavities ?" Journal of the Acoustical Society of America, vol. 80, pp. 12091214, 1986.

[2] J. D. Achenbach and M. Kitahara, "Harmonic waves in a solid with a periodic distribution of spherical cavities," Journal of the Acoustical Society of America, vol. 81, pp. 595-598, 1987.

[3] M. M. Sigalas and E. N. Economou, "Elastic and acoustic wave band structure," Journal of Sound and Vibration, vol. 158, no. 2, pp. 377-382, 1992.

[4] M. Sigalas and E. N. Economou, "Band structure of elastic waves in two dimensional systems," Solid State Communications, vol. 86, no. 3, pp. 141-143, 1993.

[5] M. S. Kushwaha, P. Halevi, G. Martínez, L. Dobrzynski, and B. Djafari-Rouhani, "Theory of acoustic band structure of periodic elastic composites," Physical Review B, vol. 49, no. 4, pp. 23132322, 1994.

[6] C.-S. Kee, J.-E. Kim, H. Y. Park, K. J. Chang, and H. Lim, "Essential role of impedance in the formation of acoustic band gaps," Journal of Applied Physics, vol. 87, no. 4, pp. 1593-1596, 2000.

[7] R. Esquivel-Sirvent and G. H. Cocoletzi, "Band structure for the propagation of elastic waves in superlattices," Journal of the Acoustical Society of America, vol. 95, no. 1, pp. 86-90, 1994.

[8] M. I. Hussein, G. M. Hulbert, and R. A. Scott, "Dispersive elastodynamics of 1D banded materials and structures: analysis," Journal of Sound and Vibration, vol. 289, no. 4-5, pp. 779-806, 2006.

[9] M. I. Hussein, G. M. Hulbert, and R. A. Scott, "Dispersive elastodynamics of 1D banded materials and structures: design," Journal of Sound and Vibration, vol. 307, no. 3-5, pp. 865-893, 2007.
[10] Y. P. Zhao and P. J. Wei, "The band gap of $1 \mathrm{D}$ viscoelastic phononic crystal," Computational Materials Science, vol. 46, no. 3, pp. 603-606, 2009.

[11] M.-L. Wu, L.-Y. Wu, W.-P. Yang, and L.-W. Chen, "Elastic wave band gaps of one-dimensional phononic crystals with functionally graded materials," Smart Materials and Structures, vol. 18, no. 11, Article ID 115013, 2009.

[12] P. D. Sesion Jr., E. L. Albuquerque, C. Chesman, and V. N. Freire, "Acoustic phonon transmission spectra in piezoelectric AlN/GaN Fibonacci phononic crystals," European Physical Journal B, vol. 58, no. 4, pp. 379-387, 2007.

[13] F.-M. Li, Y.-Z. Wang, B. Fang, and Y.-S. Wang, "Propagation and localization of two-dimensional in-plane elastic waves in randomly disordered layered piezoelectric phononic crystals," International Journal of Solids and Structures, vol. 44, no. 22-23, pp. 7444-7456, 2007.

[14] F.-M. Li, M.-Q. Xu, and Y.-S. Wang, "Frequency-dependent localization length of $\mathrm{SH}$-wave in randomly disordered piezoelectric phononic crystals,' Solid State Communications, vol.141, no. 5, pp. 296-301, 2007.

[15] Y.-Z. Wang, F.-M. Li, W.-H. Huang, and Y.-S. Wang, "The propagation and localization of Rayleigh waves in disordered piezoelectric phononic crystals," Journal of the Mechanics and Physics of Solids, vol. 56, no. 4, pp. 1578-1590, 2008.

[16] Y.-Z. Wang, F.-M. Li, K. Kishimoto, Y.-S. Wang, and W.H. Huang, "Wave localization in randomly disordered layered three-component phononic crystals with thermal effects," Archive of Applied Mechanics, vol. 80, no. 6, pp. 629-640, 2010.

[17] H. Policarpo, M. M. Neves, and A. M. R. Ribeiro, "Dynamical response of a multi-laminated periodic bar: analytical, numerical and experimental study," Shock and Vibration, vol. 17, no. 4-5, pp. 521-535, 2010.

[18] J. S. Jensen, "Phononic band gaps and vibrations in one- and two-dimensional mass-spring structures," Journal of Sound and Vibration, vol. 266, no. 5, pp. 1053-1078, 2003.

[19] G. Wang, J. Wen, Y. Liu, and X. Wen, "Lumped-mass method for the study of band structure in two-dimensional phononic crystals," Physical Review B, vol. 69, no. 18, Article ID 184302, pp. 1-184302, 2004.

[20] D. Richards and D. J. Pines, "Passive reduction of gear mesh vibration using a periodic drive shaft," Journal of Sound and Vibration, vol. 264, no. 2, pp. 317-342, 2003. 

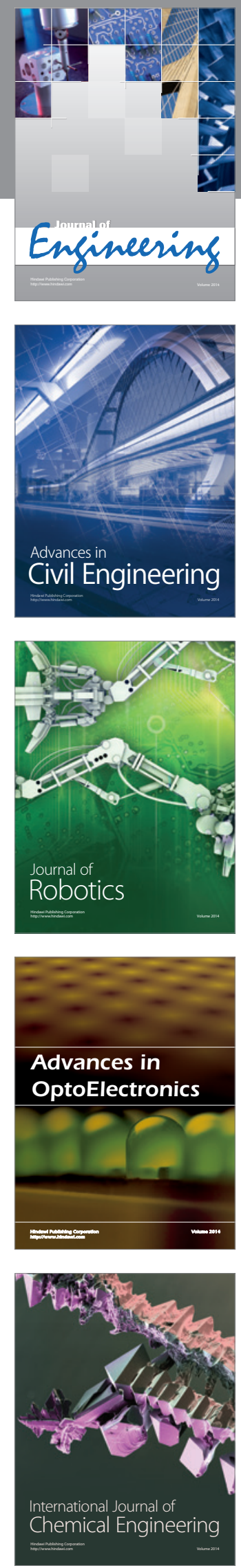

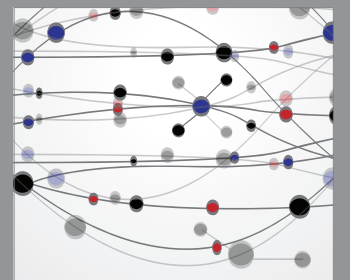

The Scientific World Journal
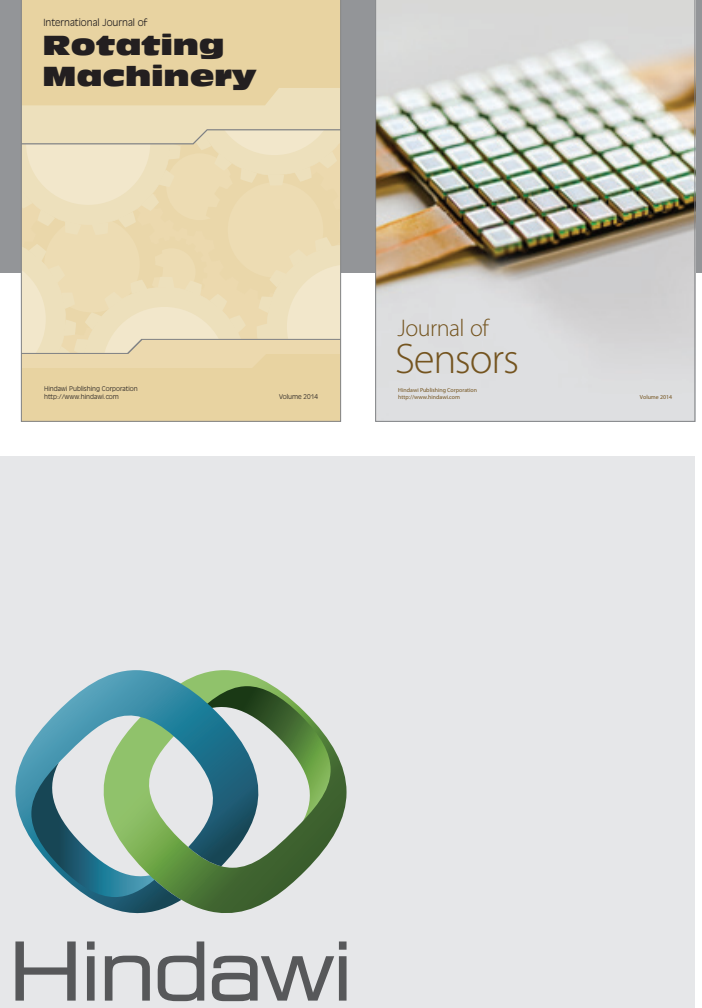

Submit your manuscripts at http://www.hindawi.com
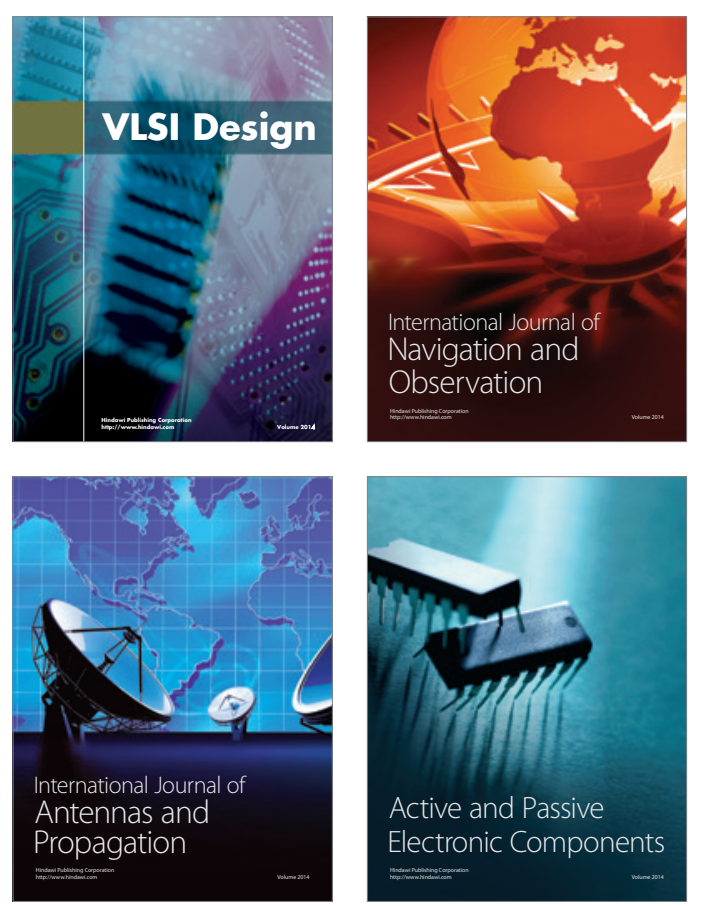
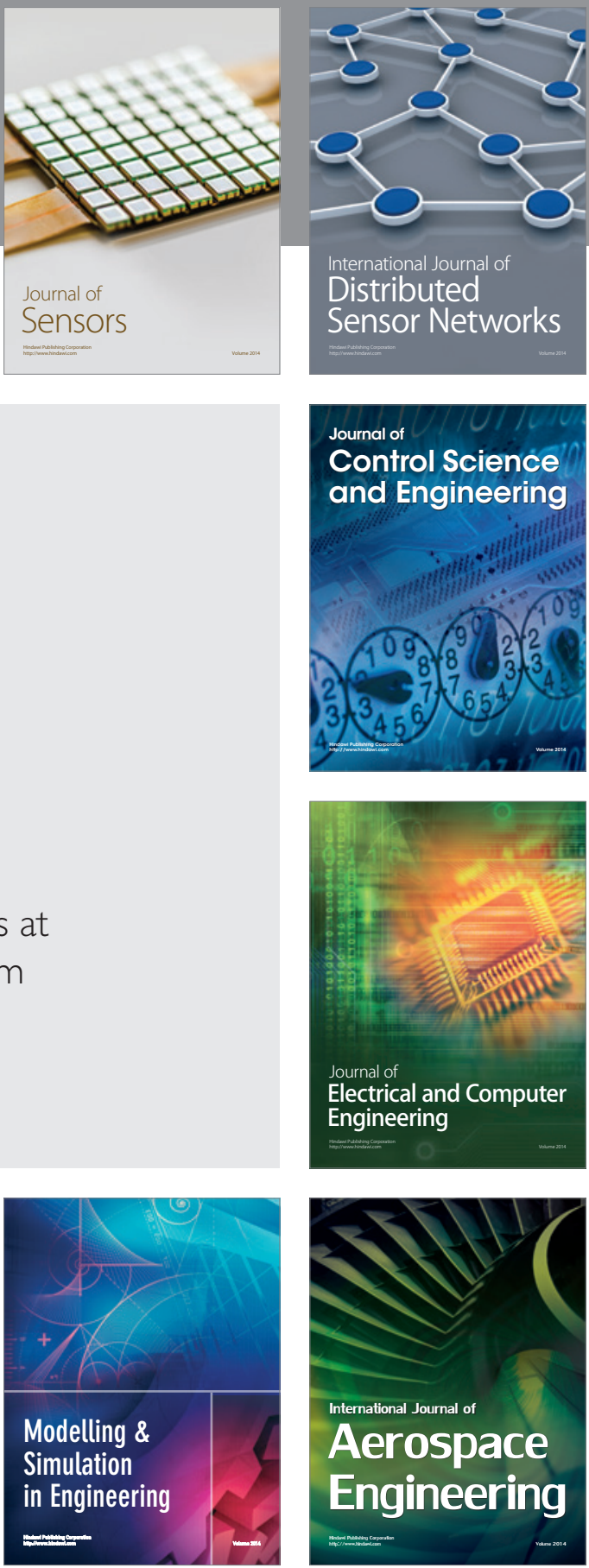

Journal of

Control Science

and Engineering
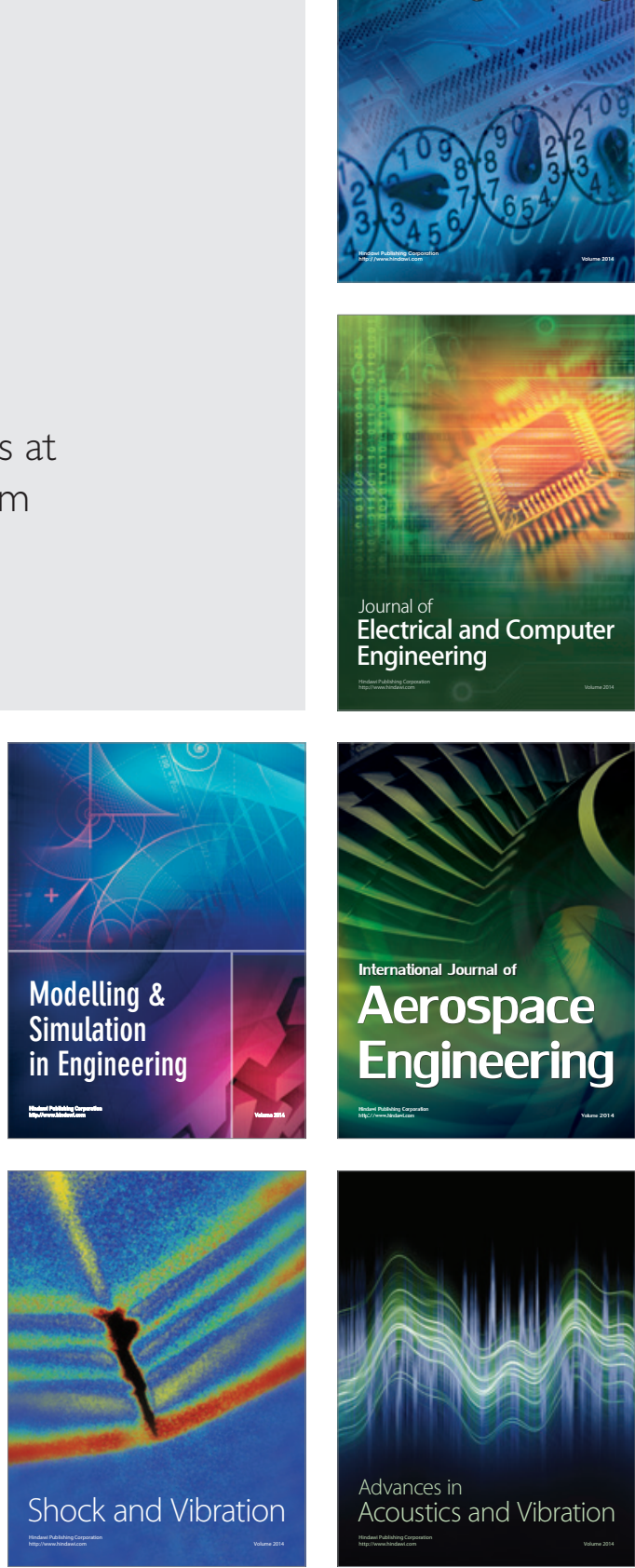